\section{BAJA PUBLICACIÓN DE INVESTIGACIONES MÉDICO ESTUDIANTILES CURRICULARES DE UNA UNIVERSIDAD PERUANA}

\author{
LOW MEDICAL STUDENT RESEARCH \\ PUBLICATIONS IN A PERUVIAN UNIVERSITY
}

\author{
Miluska O. Mejia1,a, Liz Veramendi-Espinoza ${ }^{1, a}$, \\ Yesenia M. Huerta-Collado, , \\ Juan J. Montenegro-Idrogo ${ }^{1, b}$
}

\begin{abstract}
Sr. Editor. La investigación es un elemento indispensable en la educación universitaria ${ }^{(1)}$, esta culmina por medio de publicaciones científicas, que sirven para medir esta actividad de las instituciones ${ }^{(2)}$. Es cierto que en nuestro medio, existe una pobre cultura de publicación ${ }^{(3)}$, no obstante, en los últimos años se evidencia una actividad significativa en estudiantes de Medicina humana (4). En estos reportes de pregrado lidera a nivel nacional la Facultad de Medicina de la Universidad Nacional Mayor de San Marcos ${ }^{(4)}$, quien presenta cursos en los que se realiza investigación a lo largo de su currícula ${ }^{(5)}$. No obstante, no se ha estudiado el comportamiento de la publicación de estas investigaciones desde los primeros años, como en el curso de Patología que se realiza en el segundo año de estudios y constituye uno de los que compila el mayor número de investigaciones curriculares culminadas de estudiantes de medicina en el año.
\end{abstract}

Por ello, se realizó un estudio bibliométrico descriptivo para estimar la frecuencia de publicación de los trabajos de investigación realizados entre los años 2001 y 2011. Se efectuó una búsqueda selectiva en Google Académico mediante una estrategia (4) que fue modificada por la combinación de los términos: población de estudio, blanco de estudio, agente de estudio, y efecto de estudio; seguido de la combinación de apellidos paternos de autores de la siguiente manera: (población estudio OR órgano blanco OR agente o sustancia de estudio OR efecto o resultado principal) AND (autor: Primero OR autor: Segundo OR autor: tercero). Se repitió la búsqueda cada cuatro autores y con todos en general. La búsqueda se realizó con términos en inglés y se realizó una búsqueda control para la corroboración

\footnotetext{
Sociedad Científica San Fernando, Facultad de Medicina, Universidad

Nacional Mayor de San Marcos. Lima, Perú.

a Estudiante de Medicina; ${ }^{\mathrm{b}}$ médico cirujano.

Recibido: 18-08-14 Aprobado: 20-08-14
}

Citar como: Mejia MO, Veramendi-Espinoza L, Huerta-Collado YM, Montenegro-Idrogo JJ. Baja publicación de investigaciones médicoestudiantiles curriculares de una universidad peruana. Rev Peru Med Exp Salud Publica. 2014;31(3):608. de los resultados de la primera búsqueda.

De 183 trabajos incluidos solo seis $(3,3 \%)$ fueron publicados en dos revistas locales, dos en la revista Anales de la Facultad de Medicina, y cuatro en la revista Ciencia e Investigación Médica Estudiantil Latinoamericana (CIMEL).

Este hallazgo coincide con resultados citados en estudios previos que muestran una baja tasa de publicación de investigaciones en estudiantes. Así, la baja cultura de publicación médica en Perú evidencia un comportamiento similar del encontrado en el pregrado ${ }^{(3,4)}$. Sin embargo, esta frecuencia de publicación en un curso de investigación de primeros años es menor en relación a la mayor frecuencia de publicación de un curso de quinto año de la misma universidad $(11,8 \%)$ que sugiere una tendencia mayor con el transcurso de los años, en los cuales la experiencia de trabajos realizados y de cursos de investigación es mayor ${ }^{(5)}$, siendo necesario reforzar las estrategias para la culminación efectiva de estas investigaciones en publicaciones científicas desde los primeros años.

Fuentes de financiamiento: autofinanciado.

Conflictos de interés: los autores declararán no tener conflictos de interés.

\section{REFERENCIAS BIBLIOGRÁFICAS}

1. Sedlacek S. The role of universities in fostering sustainable development at the regional level. Jour Clean Prod. 2013;48:74-84.

2. Vanclay JK, Bornmann L. Metrics to evaluate research performance in academic institutions: a critique of ERA 2010 as applied in forestry and the indirect $\mathrm{H} 2$ index as a possible alternative. Scientometrics. 2012;91:751-71.

3. Montenegro-Idrogo JJ. Huerta-Collado YM. Cultura de publicación: realidades diferentes [carta]. Rev Med Chile. 2014;142(3):402.

4. Toro-Polo LM, Pereyra-Elías R, Nizama-Vía A, Ng-Sueng LF, Vélez-Segovia E, Galán-Rodas E, et al. Publicación de los trabajos presentados a los congresos científicos de estudiantes de medicina, Perú 2002-2009: características y factores asociados. Rev Peru Med Exp Salud Publica. 2012;29(4):461-8.

5. Alarcón-Villaverde J, Romaní F, Gutiérrez C. Publicaciones científicas estudiantiles producidas en el curso de Epidemiología de la Facultad de Medicina de la Universidad Nacional Mayor de San Marcos durante el periodo 2003-2009. An Fac Med. 2010;71(2):111-6.

Correspondencia: Miluska Mejia

Dirección: Urb. Valle de naranjal Mz D Lote 22. SMP, Lima, Perú

Teléfono: +511979371543

Correo electrónico:mmejiatrebejo@gmail.com 